\title{
Preface for the Soft and Green Manufacturing and Applications
}

\author{
Paulo Bartolo ${ }^{1} \cdot$ Seung Hwan $\mathrm{Ko}^{2} \cdot \mathrm{Hugo} \mathrm{Rodrigue}^{3}$
}

Accepted: 24 March 2021 / Published online: 26 April 2021

(c) Korean Society for Precision Engineering 2021

Recent technological developments in soft devices and materials have placed a lot of focus on bringing electronic and mechanical devices closer to humans. While these devices were previously located far away from humans by operating solely in factories or within rigid boxes with which users seldom physically interacted, future devices are poised to interact much closer with humans in their everyday life. They have the potential to become healthcare assistants or domestic workers, skin-like wearable electronic components and morphing appliances, or even devices operating directly from within their body.

Devices that do not operate in direct contact with humans do not need to be designed to be inherently safe for operation around humans as rigid barriers can be built to separate them. This can include a rigid box in which electronic devices are installed or a rigid enclosure in which an industrial robot operates. These barriers can help guarantee that no accidents will occur but placing these devices closer to human requires to tackle issues related to safety and convenience of use. This can only be done by revising their core concepts and building devices that have little in common with their ancestors. Thus, the use of soft, flexible, stretchable, bio-compatible, or even edible electronic or mechanical devices is likely to become core technologies for deeper and safer interactions with humans.

The development of stretchable/flexible electronics, optics, structures, and sensors has required novel approaches

Paulo Bartolo

paulojorge.dasilvabartolo@manchester.ac.uk

$\triangle$ Seung Hwan Ko

maxko@snu.ac.kr

$\triangle$ Hugo Rodrigue

Rodrigue@skku.edu

1 The University of Manchester, Manchester, UK

2 Department of Mechanical Engineering, Seoul National University, Seoul, Republic of Korea

3 School of Mechanical Engineering, Sungkyunkwan University, Suwon, Republic of Korea at every step including new design philosophies, new material development, and novel manufacturing processes. These developments have often been guided by the needs of a wide range of applications which had not been considered previously in conventional rigid devices, and many leading research centers and companies have devoted significant effort to develop such technology and to apply them in human-friendly futuristic products. Another device for which soft replacements are being investigated is the traditional robot made from gears and rigid parts as they are bulky, heavy, and unsafe to work with humans. New actuation and sensing approaches that enable these robots to be compliant and lightweight have attracted significant attention from the public. Last, most human-made devices are made from materials that do not resemble those from which humans and animals are made, so bioprinting and biomanufacturing processes have a huge potential to directly manufacture products that are biologically safe.

This special edition will focus on the manufacturing and applications of soft and green devices to build a bridge between different technologies and promote their discussion within the scientific community. The topics being discussed in this special issue are as follows:

- Stretchable/flexible electronics, optics, structures and sensors

- Soft/green robotics and actuators

- Bio printing/manufacturing process

- Machine-robot interface

Publisher's Note Springer Nature remains neutral with regard to jurisdictional claims in published maps and institutional affiliations. 


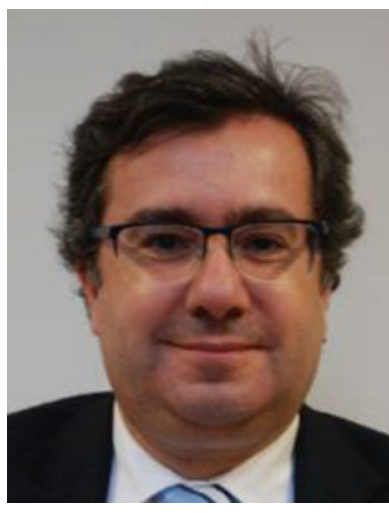

Paulo Bartolo is Chair Professor on Advanced Manufacturing at the University of Manchester (UK), where he is the Industry 4.0 Academic Lead and the Head of the Manufacturing Group. He holds a PhD in Polymer Physics from the University of Reading (UK) and an MSc and first degree in Mechanical Engineering from the University of Lisbon (Portugal). His current research interests include digital and smart manufacturing, additive manufacturing and biomanufacturing for tissue engineering

applications.

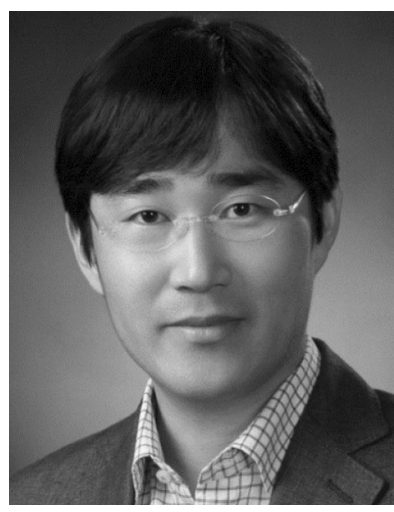

Seung Hwan Ko is a professor in Applied Nano \& Thermal Science (ANTS) Lab, mechanical Engineering department., Seoul National University, Korea. Before joining Seoul National University, he had been a faculty at KAIST, Korea since 2009. He received his $\mathrm{Ph} . \mathrm{D}$. degree in mechanical engineering from UC Berkeley in 2006. He worked as a postdoc at UC Berkeley until 2009. His current research interests are stretchable/flexible electronics, transparent electronics, soft robotics, wearable electronics, laser-assisted nano/micro fabrication, and crack assisted nanomanufacturing.

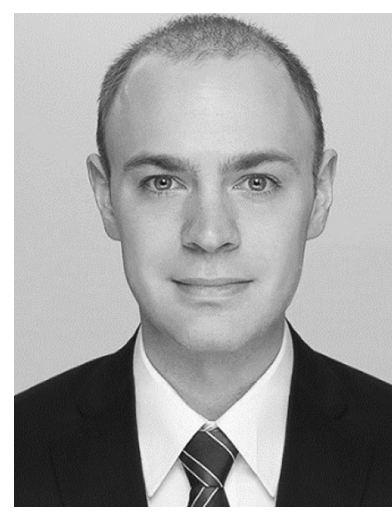

Hugo Rodrigue is an assistant professor in the School of Mechanical Engineering at Sungkyunkwan University where he leads the Soft Robotics Lab since 2016. He received his Ph.D. degree in mechanical engineering from Seoul National University in 2015, his M.S. from Ecole Polytechnique de Montreal in 2011, and his B.Eng. from McGill University in 2008. He worked as a postdoc and a Korean Research Fellow in Seoul National University from 2015 to 2016. His current research interests are soft robotics, soft pneumatic actuation, tensegrity robots, and smart materials. 